\title{
Asymmetric hydroarylation of hetero-atom containing norbornene derivatives and enantioselective synthesis of analogs of epibatidine
}

\author{
Xiao-Guang Li, ${ }^{\text {a }}$ Fang-Yi Tang, ${ }^{\mathrm{b}}$ Hua-Dong Xu, ${ }^{\mathrm{b}}$ Xin-Yan Wu ${ }^{\mathrm{b}}$ and Qi-Lin Zhou *,a \\ ${ }^{a}$ The State Key Laboratory and Institute of Elemento-organic Chemistry, \\ Nankai University, Tianjin 300071, China \\ ${ }^{b}$ Institute of Fine Chemicals, East China University of Science and Technology \\ Shanghai 200237, China \\ E-mail:qlzhou@public.tpt.tj.cn
}

\section{Dedicated to Professor Zhi-Tang Huang on the occasion of his 75th birthday} (received 18 Sep 02; accepted 04 Nov 02; published on the web 02 Dec 02)

\begin{abstract}
The reductive Heck-type hydroarylation reactions of hetero-atom containing norbornene derivatives catalyzed by palladium complexes of chiral quinolinyl-oxazolines were examined and moderate to good chiral inductions and chemical yields were achieved. Using this new method N-protected epibatidine was readily synthesized.
\end{abstract}

Keywords: Epibatidine, Heck reaction, quinolyl-oxazolines

\section{Introduction}

Epibatidine (1), a novel alkaloid, was isolated by Daly and co-workers ${ }^{1}$ from skin of Ecuadorian frog, Epipedobates tricolor in trace amounts (less than $1 \mathrm{mg}$ from 750 frogs). Because of the paucity of the natural product, the determination of its absolute stereochemistry was not investigated until 1994 by Fletcher et al. ${ }^{2}$, establishing it as $1 R, 2 R, 4 S$. It was found to be at least 200 times more potent than morphine in bioassays of analgesic-like effects in mice, but its effects were not blocked by the opiate receptor antagonist naloxone. ${ }^{1,3-5}$ Due to its outstanding pharmacological activity, unique structure, and scarcity in nature, epibatidine has been the subject of many biological ${ }^{3-11}$ and synthetic studies. ${ }^{12,13}$ The strategies reported for the synthesis of this novel alkaloid primarily focused on the synthesis of the racemic form although the optically active epibatidine can be obtained by involving an optical resolution step at an appropriate stage. ${ }^{14}$ Recently, the direct asymmetric access to this natural compound has attracted increasing attention. In 1996, Trost $^{13 a}$ reported an asymmetric synthesis of (-)-epibatidine via a 
Pd-catalyzed desymmetrization of cis-3,6-dibenzoyloxy-2-cyclohexene and a Pd-catalyzed cross coupling. Kosugi ${ }^{13 \mathrm{~b}}$ described an enantioselective procedure based on the asymmetric protonation of achiral lithium enolates derived from cyclohexanone derivatives with chiral $\beta$ hydroxy sulfoxides in 1997. Kibayashi ${ }^{13 e}$ achieved the asymmetric access by using asymmetric hetero Diels-Alder cycloaddition of chiral N-acylnitroso dienophile. However, these methods lack easy adaptability for the synthesis of optically active epibatidine due to too many steps and low overall chemical yields. In 1999, Kaufmann ${ }^{13 \mathrm{~g}}$ reported an enantioselective synthesis of Nprotected epibatidine via Pd-catalyzed asymmetric Heck-type hydroarylation of azabicycles with 2-chloro-5-iodopyridine using BINAP ligands. In his process the coupling reaction, the key step to control enantioselectivity, does not occur until the penultimate step of the synthesis of epibatidine. Although the chemical yield and the enantioselectivity were not high in Kaufmann's route the asymmetric hydroarylation reaction was shown to be a promising tool in the synthesis of epibatidine. It is obviously important to find a proper chiral ligand for the improvement of efficiency of catalyst in the hydroarylation reaction.

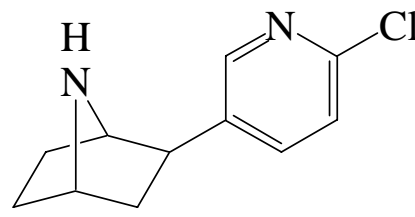

1

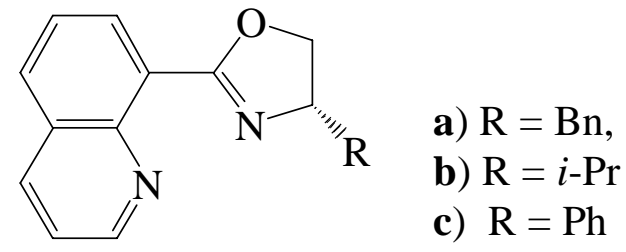

2

\section{Results and Discussion}

In the previous work, we have demonstrated that the chiral quinolinyl-oxazolines 2 were efficient ligands in Pd-catalyzed asymmetric Heck-type hydroarylation of norbornene and its derivatives. ${ }^{15}$ It is of interest to study the application of this type of dinitrogen ligands in the enantioselective synthesis of epibatidine and its analogs. Moreover, many norbornene derivatives containing nitrogen or oxygen atoms were reported to have important biological activities. ${ }^{9}$ In this paper, we would like to describe our results in the investigations on the palladium catalyzed reductive Heck-type hydroarylation of hetero-atom containing norbornene derivatives using chiral ligands 2 and the asymmetric synthesis of $\mathrm{N}$-protected epibatidine as shown in Scheme 1.

The hydroarylation reactions were carried out in DMSO, $\mathrm{HCOOH}$ was used as a hydride source and the catalysts (5 mol\%) were prepared in situ from $\left[\mathrm{Pd}(\mathrm{dba})_{2}\right]$ and ligand $2 \mathbf{a}$. 


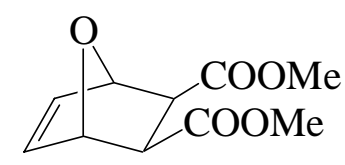

3

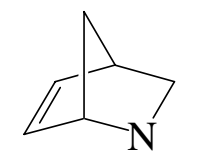

4

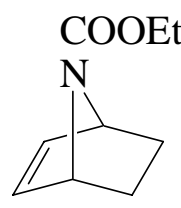

ArI / Pd / 2a

$\mathrm{HCOOH} /$ base

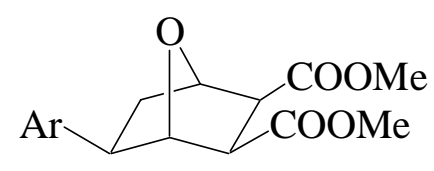

6a: $\mathrm{Ar}=\mathrm{Ph}$

6b: $\mathrm{Ar}=p-\mathrm{MeOPh}$

6c: $\mathrm{Ar}=p-\mathrm{MePh}$
Boc

5

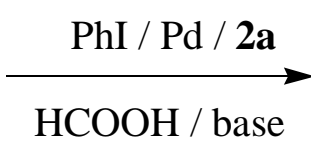

$\mathrm{HCOOH} /$ base

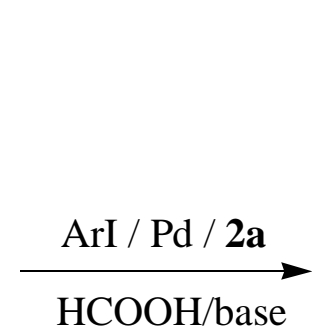

HCOOH/base

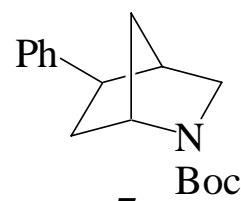

7

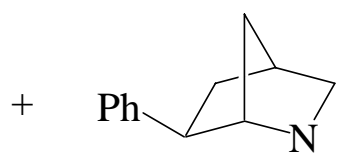

8 Boc

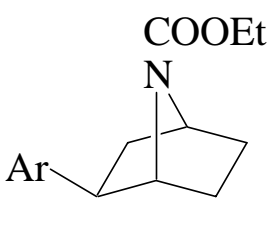

9a: $\mathrm{Ar}=\mathrm{Ph}$

9b: Ar $=6-\mathrm{Cl}-3-\mathrm{Py}$

\section{Scheme 1}

Three types of substrate containing nitrogen or oxygen atoms were examined and the results are summarized in Table 1. In the case of substrate 3, moderate enantioselectivities were achieved. Proton sponge and diisopropylethylamine were found to be efficient bases in the hydroarylation of 3, while triethylamine gave lower enantiomeric excess. Introduction of electron-donating groups on the phenyl ring of phenyl iodide had a very weak influence on the enantioselectivity. In the hydroarylation of $\mathbf{4}$, two isomers $\mathbf{7}$ and $\mathbf{8}$ were isolated, and compound $\mathbf{8}$ was obtained in higher ee. The reaction of $\mathbf{4}$ was quite slow and the yields of hydroarylation products were low, which could be improved by using more catalyst (10 mol\%) (entry 7 vs 6).

Finally, the hydroarylation of azabicyclo[2,2,1]hept-2-ene (5) was examined under the same conditions. Encouraged by the result of the reaction with phenyl iodide (65\% ee in $75 \%$ yield), the hydroarylation of $\mathbf{5}$ with 2-chloro-5-iodopyridine was performed. The desired product, $\mathbf{N}$ protected epibatidine $\mathbf{9 b}$, was obtained in $67 \%$ yield with $51 \%$ ee that provided one of few examples of catalytic enantioselective synthesis of epibatidine and its analogs.

The methodology outlined here on the asymmetric hydroarylation of hetero-atom containing norbornene derivatives should open a new access to epibatidine and its analogs.

Table 1. Asymmetric reductive Heck-type hydroarylation of hetero-atom containing norbornene derivatives $^{\text {a }}$ 


\begin{tabular}{|c|c|c|c|c|c|c|c|}
\hline Entry & Substrate & ArI & Base & Time $^{b}$ & Product & Yield (\%) ${ }^{c}$ & Ee $(\%)^{d}$ \\
\hline 1 & 3 & $\mathrm{PhI}$ & $i-\mathrm{Pr}_{2} \mathrm{NEt}$ & $20 \mathrm{~h}$ & $6 a$ & 68 & 68 \\
\hline 2 & 3 & $\mathrm{PhI}$ & $\mathrm{NEt}_{3}$ & $24 \mathrm{~h}$ & $\mathbf{6 a}$ & 87 & 49 \\
\hline 3 & 3 & $\mathrm{PhI}$ & $\mathrm{PS}^{\mathrm{e}}$ & $24 \mathrm{~h}$ & $\mathbf{6 a}$ & 87 & 65 \\
\hline 4 & 3 & $p$-MeOPhI & $i-\mathrm{Pr}_{2} \mathrm{NEt}$ & $36 \mathrm{~h}$ & $6 \mathbf{b}$ & 55 & 66 \\
\hline 5 & 3 & p-MePhI & $i-\mathrm{Pr}_{2} \mathrm{NEt}$ & $36 \mathrm{~h}$ & $6 c$ & 57 & 60 \\
\hline 6 & 4 & PhI & $i-\mathrm{Pr}_{2} \mathrm{NEt}$ & 7 days & $7+8$ & $43(43 / 57)$ & $59 / 82$ \\
\hline $7^{\mathrm{f}}$ & 4 & PhI & $i-\mathrm{Pr}_{2} \mathrm{NEt}$ & 7 days & $7+8$ & $71(46 / 54)$ & $70 / 85$ \\
\hline 8 & 5 & PhI & $i-\mathrm{Pr}_{2} \mathrm{NEt}$ & $48 \mathrm{~h}$ & $9 a$ & 75 & 65 \\
\hline 9 & 5 & $\mathrm{CIP}^{\mathrm{g}}$ & $i-\mathrm{Pr}_{2} \mathrm{NEt}$ & 4 days & $9 b$ & 67 & 51 \\
\hline $\mathrm{TH}$ & reaction & were & erformed & DMS & at & ${ }^{\circ} \mathrm{C}$. & ents: \\
\hline
\end{tabular}

$\left[\mathrm{Pd}(\mathrm{dba})_{2}\right] / 2 a /$ Substrate/ArI/HCOOH/ Base $=0.05 / 0.12 / 1 / 3 / 3 / 3.5 \mathrm{mmol}$, unless mentioned otherwise. ${ }^{b}$ Time for complete conversion of substrate or appearance of palladium black.

${ }^{C}$ Isolated yield. ${ }^{d}$ Determined by HPLC (for 6a: Chiracel OD-R, $\mathrm{MeOH} / \mathrm{H}_{2} \mathrm{O}=90: 10,1.0$ $\mathrm{ml} / \mathrm{min}$; for 6b: Chiracel OD, $n$-hexane/2-PrOH = 90:10, $1.0 \mathrm{ml} / \mathrm{min}$; for $6 \mathbf{c}$ : Chiracel OD, $n$ hexane/PrOH = 93:7, $0.6 \mathrm{ml} / \mathrm{min}$. for 7 and 8: Chiracel OD, $n$-hexane/PrOH = 99:1, 1.0 $\mathrm{ml} / \mathrm{min}$; for 9a and 9b: Chiracel OJ, $n$-hexane/PrOH $=90: 10,1.0 \mathrm{ml} / \mathrm{min}) .{ }^{e}$ Proton sponge [1,8-bis(dimethylaminonaphthalene)]. ${ }^{f} 10 \mathrm{~mol} \%$ of catalyst was used. ${ }^{g} \mathrm{CIP}=2$-chloro-5iodopyridine.

\section{Experimental Section}

\section{General procedure for enantioselective hydroarylation reactions}

Under an argon atmosphere, $\left[\mathrm{Pd}(\mathrm{dba})_{2}\right](0.05 \mathrm{mmol})$ and the ligand 2a $(0.12 \mathrm{mmol})$ were dissolved in dry DMSO (5 ml) and stirred at $25{ }^{\circ} \mathrm{C}$ for $2 \mathrm{~h}$. A mixture of base (3.5 mmol), formic acid (3.0 mmol), substrate $(1.0 \mathrm{mmol})$ and arylating reagent $(3.0 \mathrm{mmol})$ in dry DMSO (3 ml) was added rapidly in one portion. After stirring at the corresponding temperature until complete conversion of the substrate, the reaction mixture was distributed between water and ethyl acetate. The aqueous layer was extracted three times with ethyl acetate. The organic phase was washed with brine and dried over anhydrous magnesium sulfate. After evaporation of solvent, the residue was purified by flash column chromatography on silica gel to give products.

(6a): ${ }^{16}$ White solid, m.p. 107-110 ${ }^{\circ} \mathrm{C} .{ }^{1} \mathrm{H}$ NMR (300 MHz, $\left.\mathrm{CDCl}_{3}\right): \delta$ 7.15-7.30 (m, 5H), 5.06 (d, $\mathrm{J}=5.4 \mathrm{~Hz}, 1 \mathrm{H}$ ), 4.84 (s, 1H), 3.70 (s, 3H), 3.67 (s, 3H), 3.12 (dd, J = 21.3, 9.6 Hz, 2H), 2.93 (dd, $\mathrm{J}=9.0,5.1 \mathrm{~Hz}, 1 \mathrm{H}), 2.15$ (dd, $\mathrm{J}=12.9,9.0 \mathrm{~Hz}, 1 \mathrm{H}), 1.82-1.92(\mathrm{~m}, 1 \mathrm{H})$.

(6b): White solid, m.p. $122-125{ }^{\circ} \mathrm{C} .{ }^{1} \mathrm{H}$ NMR (300 MHz, $\mathrm{CDCl}_{3}$ ): $\delta 7.20$ (dd, J = 6.6, $2.1 \mathrm{~Hz}$, 2H), 6.82 (dd, J = 6.6, $2.1 \mathrm{~Hz}, 2 \mathrm{H}$ ), 5.04 (d, J = 5.4 Hz, 1H), 4.78 (s, 1H), 3.70 (s, 3H), 3.70 (s, 3H), 3.67 (s, 3H), 3.10 (dd, J = 20.7, $9.6 \mathrm{~Hz}, 2 \mathrm{H}$ ), 2.89 (dd, J = 9.0, $5.1 \mathrm{~Hz}, 1 \mathrm{H}$ ), 2.13 (dd, J = 12.9, $9.0 \mathrm{~Hz}, 1 \mathrm{H}), 1.78-1.88$ (m, 1H). IR (KBr): 3005w, 2970w, 1740s, 1730s, 1610s, 1460m, 
1420m, 1360m, 1300s, 1220s, 1180s. MS (EI): 320 (32, M+), 218 (88), 159 (73), 147 (72), 121 (100). Anal. calcd for $\mathrm{C}_{17} \mathrm{H}_{20} \mathrm{O}_{6}$ : C, 63.74; H, 6.29. Found: C, 63.50; H, 6.05.

(6c): White solid, m.p. 124-126 ${ }^{\circ} \mathrm{C} .{ }^{1} \mathrm{H}$ NMR (300 M Hz, $\left.\mathrm{CDCl}_{3}\right): \delta 7.17$ (d, J = $6.0 \mathrm{~Hz}, 2 \mathrm{H}$ ), 7.09 (d, J = 8.1 Hz, 2H), 5.04 (d, J = $5.1 \mathrm{~Hz}, 1 \mathrm{H}), 4.80$ (s, 1H), 3.70 (s, 3H), 3.66 (s, 3H), 3.11 (dd, J = 21.3, 9.6 Hz, 2H), 2.90 (dd, J = 9.0, $5.1 \mathrm{~Hz}, 1 \mathrm{H}$ ), 2.31 (s, 3H), 2.13 (dd, J = 12.9, $9.0 \mathrm{~Hz}$, $1 \mathrm{H}), 1.80-1.90$ (m, 1H). IR (KBr): 2950w, 1748s, 1735s, 1434m, 1350m, 1218s, 1194s. MS (EI): 304 (21, $\mathrm{M}^{+}$), 202 (38), 143 (100), 105 (38). Anal. calcd for $\mathrm{C}_{17} \mathrm{H}_{20} \mathrm{O}_{5}$ : C, 67.09; H, 6.62. Found: C, 67.35; H, 6.51.

(7+8): ${ }^{12 \mathrm{e}}$ Colorless oil. ${ }^{1} \mathrm{H}$ NMR (300 MHz, $\left.\mathrm{CDCl}_{3}\right): \delta 7.15-7.30$ (m, 5H), 4.10-4.40 (4s, $\left.1 \mathrm{H}\right)$, 2.98-3.40 (m, 3H), 2.65 (s, 1H), 2.10-2.40 (m, 1H), 1.90-1.97 (m, 1H), 1.58-1.64 (m, 2H), 1.48$1.51(2 \mathrm{~s}, 9 \mathrm{H})$.

(9a): ${ }^{13 g}$ Colorless oil. ${ }^{1} \mathrm{H}$ NMR (300 $\mathrm{MHz}, \mathrm{CDCl}_{3}$ ): $\delta$ 7.15-7.25 (m, 5H), 4.25-4.50 (m, 2H), 4.00-4.20 (br. s, 2H), 2.80-2.95 (m, 1H), 1.50-2.00 (m, 6H), 1.05-1.25 (br. s, 3H).

(9b): ${ }^{13 g}$ Colorless oil. ${ }^{1} \mathrm{H}$ NMR (200 MHz, $\mathrm{CDCl}_{3}$ ): $\delta 8.25$ (s, $\left.1 \mathrm{H}\right), 7.68$ (d, J = $\left.8.1 \mathrm{~Hz}, 1 \mathrm{H}\right), 7.25$ (d, J = 8.1 Hz, 1H), 4.45-4.50 (br. s, 2H), 4.25-4.40 (br.s, 2H), 2.90 (dd, J = 8.8, $4.7 \mathrm{~Hz}, 1 \mathrm{H}$ ), 2.00-2.05 (m, 1H), 1.75-1.95 (m, 3H), 1.50-1.70 (m, 2H), 1.10-1.30 (br. s, 3H).

\section{Acknowledgements}

Financial supports from the National Natural Science Foundation of China, the Major State Basic Research Development Program (grant No. G2000077506) and the Natural Science Foundation of Tianjin are gratefully acknowledged.

\section{References}

1. Spande, T. F.; Garraffo, H. M.; Edwards, M. W.; Yeh, H. J. C.; Pannel, L.; Daly, J. W. J. Am. Chem. Soc. 1992, 114, 3475.

2. Fletcher, S. R.; Baker, R.; Chambers, M. S.; Herbert, R. H.; Hobbs, S. C.; Thomas, S. R.; Verrier, H. M.; Watt, A. P.; Ball, R. B. J. Org. Chem. 1994, 59, 1771.

3. Li, T.; Qian, C.; Eckman, J.; Huang, D. F.; Shen, T. Y. Bioorg. Med. Chem. Lett. 1993, 3, 2759.

4. Qian, C.; Li, T.; Shen, T. Y.; Libertine-Garaham, L.; Eckman, J.; Biftu, T.; Ip, S. Eur. J. Pharmacol. 1993, 250, R13.

5. Badio, B.; Daly, J. W. Mol. Pharmacol. 1994, 45, 563.

6. Lippiello, P. M.; Bencherif, M.; Gray, J. A.; Peters, S.; Grigoryan, G.; Hodges, H.; Collins, A. C. J. Pharmacol. Exp. Ther. 1996, 279, 1422.

7. Meyer, E. M.; De Fiebre, C. M.; Hunter, B. E.; Simpkines, C. E.; Frauworth, N.; De Fiebre, N. E. C. Drug Dev. Res. 1994, 31, 127. 
8. Menzaghi, F.; Whelan, K. T.; Risbrough, V. B.; Rao, T. S.; Lloyd, G. K. J. Pharmacol. Exp. Ther. 1997, 280, 393.

9. (a) Javic, M. E. Br. J. Addict. 1991, 86, 571. (b) Szántay, C.; Kardos-Balogh, Z.; Szántay, Jr., C. The Alkaloids 1995, 46, 95.

10. Damaj, M. I.; Creasy, K. R.; Grove, A. D.; Rosecrans, J. A.; Martin, B. R. Brain Res. 1994, 664, 34.

11. Bannon, A. W.; Decker, M. W.; Holladay, M. W.; Curzon, P.; Donnelly-Roberts, D.; Puttfarken, P. S.; Bitner, R. S.; Diaz, A.; Dickenson, A. H.; Porsolt, R. D.; Williams, M.; Arneric, S. P. Science 1998, 279, 77.

12. (a) Clayton, S. C.; Regan, A. C. Tetrahedron Lett. 1993, 34, 7493. (b) Chen, Z.; Trudell, M. L. Chem. Rev. 1996, 96, 1179. (c) Bai, D.-L.; Xu, R.; Chu, G.-H.; Zhu, X.-Z. J. Org. Chem. 1996, 61, 4600. (d) Giblin, G. M. P.; Jones, C. D.; Simpkins, N. S. Synlett 1997, 589. (e) Cox, C. D.; Malpass, J. R. Tetrahedron 1999, 55, 11879. (f) Namyslo J. C.; Kaufmann, D. E. Synlett 1999, 114.

13. (a) Trost, B. M.; Cook, G. R. Tetrahedron Lett. 1996, 37, 7485. (b) Kosugi, H.; Abe, M.; Hatsuda, R.; Uda, H.; Kato, M. Chem. Commun. 1997, 1857. (c) Albertini, E.; Barco, A.; Benetti, S.; De Risi, C.; Pollini, G. P.; Zanirato, V. Tetrahedron 1997, 53, 17177. (d) Jones, C. D.; Simpkins, N. S.; Giblin, G. M. P. Tetrahedron Lett. 1998, 39, 1023. (e) Aoyagi, S.; Tanaka, R.; Naruse, M.; Kibayashi, C. J. Org. Chem. 1998, 63, 8397. (f) Pandy, G.; Bagul, T. D.; Sahoo, A. K. J. Org. Chem. 1998, 63, 760. (g) Namyslo J. C.; Kaufmann, D. E. Synlett 1999, 804.

14. (a) Huang, D. F.; Shen, T. Y. Tetrahedron Lett. 1993, 34, 4477; (b) Corey, E. J.; Loh, T.-P.; AchyuthaRao, S.; Daly, D. C.; Sarshar, S. J. Org. Chem. 1993, 58, 5600. (c) Huang, D. F.; Shen, T. Y. Tetrahedron Lett. 1993, 34, 4477.

15. (a) Wu, X.-Y.; Xu, H.-D.; Zhou, Q.-L.; Chan, A. S. C. Tetrahedron: Asymmetry 2000, 11, 1255. (b) Wu, X.-Y.; Xu, H.-D.; Tang, F.-Y.; Zhou, Q.-L. Tetrahedron: Asymmetry 2001, 12, 2565.

16. Arcadi, A.; Marinelli, F.; Bernocchi, E.; Cacchi, S.; Ortar, G. J. Organomet. Chem. 1989, 368, 249. 